\title{
Profile characterization of diffraction gratings using angle-resolved polarimetric measurements
}

\author{
M. Foldyna ${ }^{1}$, A. De Martino ${ }^{1}$, C. Licitra ${ }^{2}$, J. Foucher ${ }^{2}$ and S. Ben Hatit ${ }^{1}$ \\ ${ }^{1}$ LPICM, CNRS, Ecole Polytechnique, 91128 Palaiseau, France \\ ${ }^{2}$ CEA LETI-MINATEC, F38054 Grenoble Cedex 9, France
}

\begin{abstract}
Development of the characterization tools and techniques used by semi-conductor industry directs not only towards increasing of the instrumental precision, to push limits of the optical characterization methods to the smallest lines, but also towards a decreased dependency of the used tools on the tool-to-tool calibration procedures. The challenge approached in this work is to use multiple independent optical configurations to determine overall accuracy of the results with minimal or no assistance of the other non-optical methods. The approach presented here is based on a well-known change of sensitivity of the optical model parameters at the different azimuthal measurement configurations. The full potential of this method can only be unlocked using complete Mueller matrix measurements providing complete information on the anisotropic nature of the gratings. The measurements at multiple azimuth configurations, used in this work, illustrate the potential of the method on the experimental data provided by the angle-resolved and spectrally resolved Mueller matrix polarimetric tools. The results are consistent with the single-line AFM measurements used as an independent reference.
\end{abstract}

\section{Introduction}

Optical characterization methods are used as one of the standards of process control in the semiconductor devices production. The main application is the determination or verification of the critical dimensions, with the line width of the produced gratings in the first place. However, optical methods are usually calibrated and their accuracy is evaluated by comparison with other independent methods, such as state-of-the-art scanning electron microscopy (SEM) and atomic force microscopy (AFM), which provide adequate information about the detailed profiles of the grating lines [1-3].

There is an increasing need to evaluate the accuracy of optical (and other metrological) methods [4] without using time-consuming AFM or destructive cross-section SEM methods, whose costs are steeply increasing with the wafer sizes. As the grating profiles obtained by fitting optical data cannot be fully independent from the initial choice of the possibilities - that is, the type of the profile has to be chosen in advance - no reliable information about the accuracy of the result can be retrieved from any single measurement and fit. To evaluate the accuracy of the result, an independent measure of the consistency of the result with the physical reality is needed. One possible method to specify the accuracy of the model is to compare and/or combine different measurement configurations. For the gratings considered here, the simplest way to achieve this is to rotate the sample and measure the grating in different, conical, configurations, which provide new and independent information about the structure [5-6].

In Section 2 the angle-resolved Mueller matrix polarimeter using microscope objectives is introduced. The operation of the experimental setup is briefly described and an example of the measured data is shown together with results of characterization of the photoresist grating. In the following Section 3 we introduce the efficient method combining spectrally resolved data in multi-azimuth configuration. A statistical method allowing determining realistic confidence intervals for all critical dimensions is also provided in this section. Section 4 introduces filtering of non-depolarizing Mueller matrix from measured depolarizing matrices. Method is tested on the boundary between the grating and substrate.

\section{Angle-resolved Mueller matrix polarimetry using microscope objective}

Mueller matrix polarimetry is an efficient method to measure and characterize optically anisotropic samples such as diffraction gratings. Complete information provided by Mueller matrices can be used to analyze depolarization effects (if present), but it also gives complete description of cross-polarizing effects, which 
are naturally occurring in diffraction gratings measured in conical configurations. High level of redundancy in the data in non-depolarizing cases gives measurements increased robustness and it can be used for the measurement accuracy analysis.

In order to be able to characterize samples small than few tens of micrometers, we are using a microscope objective which focuses incident light beam onto the sample. Resulting Mueller matrices measured using polarization state generator (PSG) and analyzer (PSA) can be achieved with help of very general eigenvalue calibration method [7]. The method, generalized for the purpose of measurements using the microscope objective, enables to avoid complications with modelling of optical components (e.g. beam splitter) and extend the measurements into multiple wavelengths [8].

Previously described experimental setup enables measuring of angle-resolved Mueller matrices as shown in Fig. 1 simply by taking sixteen subsequent images by CCD camera for different PSG and PSA configurations.

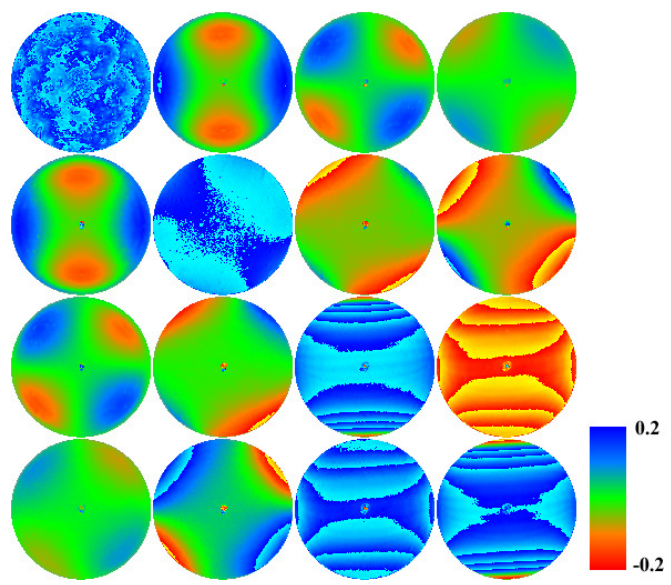

Fig. 1. Sixteen normalized Mueller matrix elements measured on photoresist grating and plotted in polar coordinates as function of polar and azimuthal angles.

The angle-resolved polarimetric images can be used to determine critical dimensions of the grating profile with high sensitivity to the profile asymmetry and sidewall angle. The result of one such characterization is shown in Fig. 2, where the acquired trapezoidal profile is directly plotted into the SEM cross-section image. The correspondence between SEM and polarimetric profiles is very good, which is due to the full use of all available conical configurations. Details of the work were published elsewhere [9].

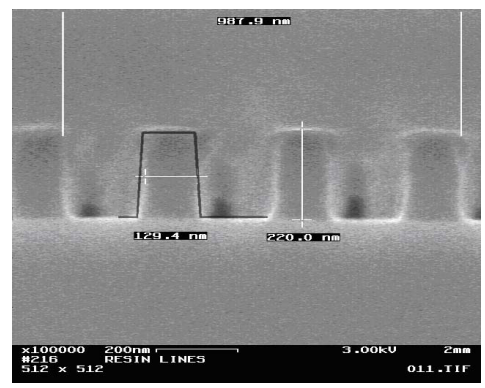

Fig. 2. SEM image of the grating cross-section compared with the optically resolved grating profile.

\section{Multi-azimuth spectrally resolved Mueller matrix polarimetry}

The previously demonstrated advantages from using conical configurations are only stronger when combined with spectrally resolved measurements. The robustness of the spectrally resolved Mueller matrix polarimetry used in multiple-azimuth configuration is demonstrated on the characterization of small pitch gratings filling $250 \mu \mathrm{m}$ wide square boxes. Used Mueller matrix polarimeter was directly installed in the clean room and had motorized rotating stage allowing the access to an arbitrary conical configuration. The projected beam spot size could be reduced to $60 \times 25 \mu \mathrm{m}$, but for the purposes of this work $100 \times 100 \mu \mathrm{m}$ spot was used. Figure 3 shows for the purposes of comparison almost identical data acquired using smaller 60x25 $\mu \mathrm{m}$ and larger 100x100 $\mu \mathrm{m}$ spots.
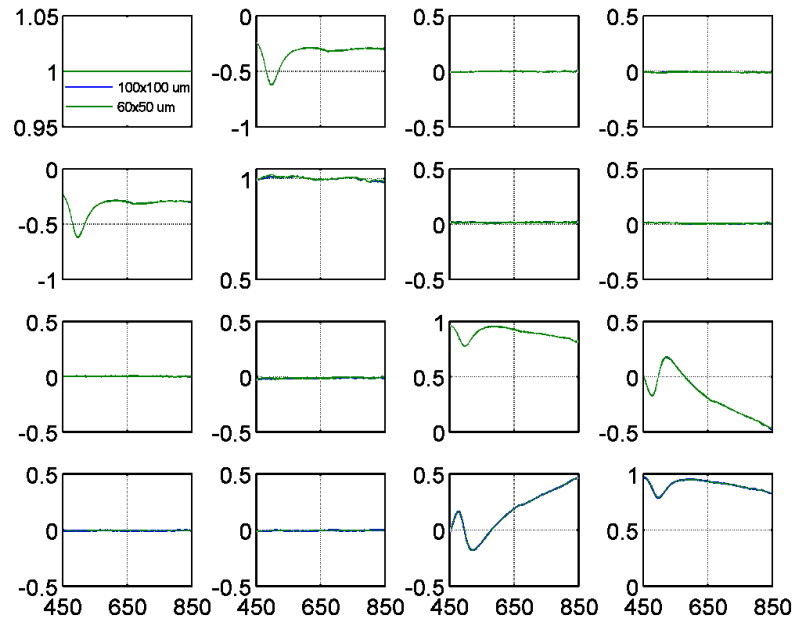

Fig. 3. Spectrally resolved Mueller matrices measured using 100x100 $\mu \mathrm{m}$ (blue curves) and 60x50 $\mu \mathrm{m}$ (green curves) spot sizes.

The optimal values of parameters of a trapezoidal profile model (see Fig. 4), acquired for each azimuthal angle separately using a non-linear least-square minimization algorithm, are shown for a typical $140 \mathrm{~nm}$ pitch grating in Fig. 5.

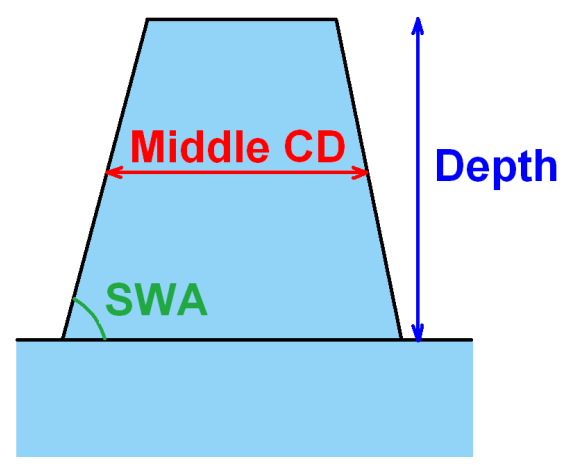

Fig. 4. Critical dimensions of trapezoidal profile used for the modeling and the grating characterization.

Small boxes in Fig. 5 representing results of the fits are surrounded by $2 \sigma$ confidence intervals falling into subnanometer and sub-degree scales. Unfortunately, these 
confidence intervals, provided from the standard analysis for each azimuth separately, are too small when compared to the dispersion of values over azimuths and cannot be considered as a realistic estimation of the accuracy of result. This strong underestimation of the confidence intervals is due to systematic measurement errors and imperfect model which does not exactly correspond to the real profile. In order to improve the correspondence of the model with real gratings, more parameters has to be included (providing more complex profiles). Introducing more profile parameters, on the other hand, negatively influences correlations between parameters and increases dependence of the results on the initial guess [6].

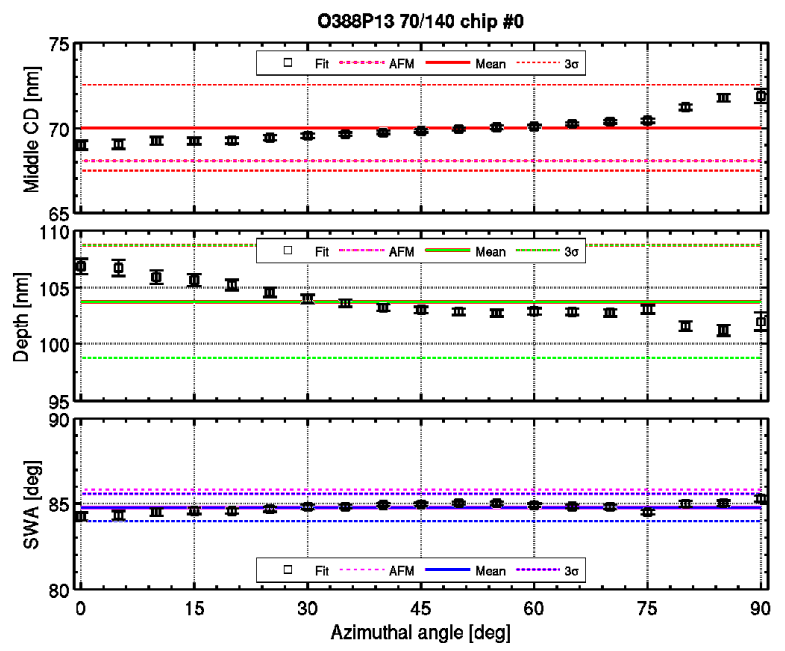

Fig. 5. Azimuthal dependence of the critical grating profile parameters acquired from separate fits. Mean values and $3 \sigma$ confidence intervals are plotted as colorful lines. Values provided by 3D-AFM reference tool are marked by dashed violet line.

Further statistical analysis of the azimuth-dependent dimensional parameters from Fig. 5 may provide realistic estimates of the confidence interval giving direct information about the accuracy of the results. The essential conclusion of our work is that the 3D-AFM values mostly fall into the confidence intervals provided by the optical method (tested on 21 different grating boxes), which means that we can estimate the accuracy of our results without using direct comparison with another, non-optical, method [10]. Moreover, this approach may provide a way to improve the accuracy of the grating profile modeling by minimizing the standard deviations evaluated from multiple-azimuths results.

\section{Characterization of gratings using depolarizing Mueller matrices}

When two non-depolarizing materials are measured using incoherent light illuminating both materials at the same time, the resulting Mueller matrix is a depolarizing superposition of the two non-depolarizing Mueller matrices corresponding to each material. Under specific conditions, the original non-depolarizing components of the measured Mueller matrix can be retrieved [11-12]. It

was recently shown that normalized Mueller matrices are sufficient for characterization of the structure when two incoherent signals from non-depolarizing materials are present [13]. When one of the two non-depolarizing components is sufficiently known and can be modelled (or its absolute reflectivity can be measured), the optical characterization of the sample from the second component can be successful. Provided the sample provides at least $15 \%$ of the overall measured intensity, the accuracy of the characterization can be equal to that obtained from the non-depolarizing Mueller matrices measured directly inside the sample [13].

More recently, a further step was reported [14] in the depolarizing Mueller matrices treatment allowing retrieval of one of the two non-depolarizing component. In practical situation this means to somehow acquire measured Mueller matrix of one of the component (e.g. by measuring it on non-patterned area) and subsequently retrieve the missing second component. The advantage of this procedure with respect to the previously mentioned method is that neither the detailed component structure nor its measured reflectivity is needed.

Normalized spectrally resolved Mueller matrices were measured on the boundary between the grating and the substrate and on each material separately. Measured depolarizing matrices from the boundary and substrate matrices are shown in Fig. 6.
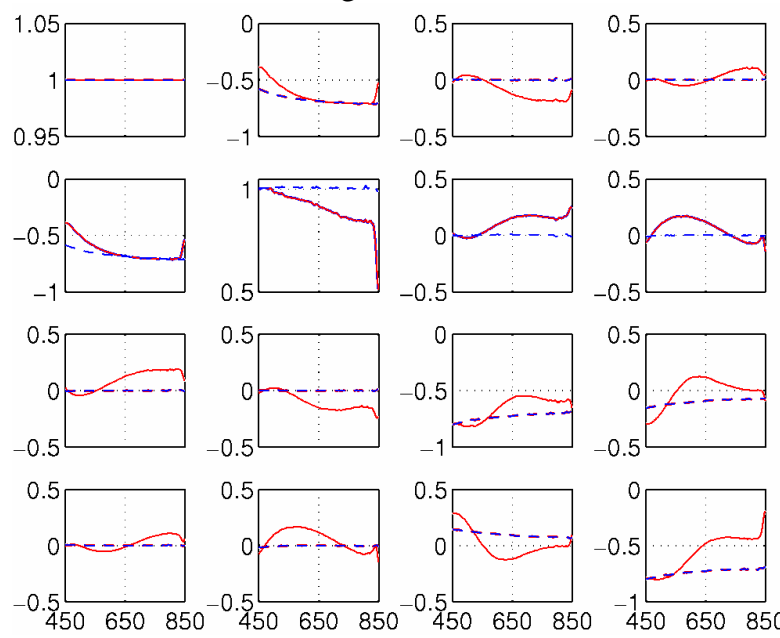

Fig. 6. Spectrally resolved Mueller matrices measured on the boundary between grating and substrate (red solid curves) and the silicon substrate (blue dashed curves).

In Fig. 7 we show the spectral dependence of eigenvalues of the coherency matrices corresponding to measured depolarizing matrices from the boundary (see Fig. 6). These matrices have two non-zero eigenvalues, which proves that the rank of this matrix is equal to two (two non-depolarizing components are present) as expected. It is worth to note that there is a narrow spectral region around $525 \mathrm{~nm}$ in Fig. 7 where values of one of non-null eigenvalues become very small. This is not without some consequences in terms of the accuracy of the retrieval of the grating's Mueller matrix.

Using the numerical and analytical approach [14] we have retrieved pure non-depolarizing Mueller matrix component of the grating from the depolarizing data from 
the boundary and compared it with the previously measured grating data from inside of the box. The resulting matrices are plotted in Fig. 8 and compared with the experimental matrix of the grating measured alone, which was marked by black solid line.

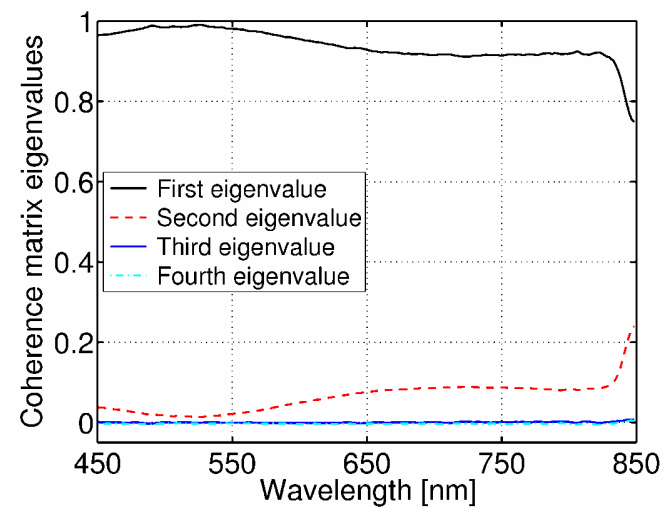

Fig. 7. Spectral dependence of eigenvalues of coherency matrices (corresponding to data in Fig. 6).

The overall correspondence between measured and numerically reconstructed data in Fig. 8 is very good apart from some slight differences around wavelength of 700 $\mathrm{nm}$ in the element $\mathrm{M}_{44}$. Differences for longer wavelengths around Rayleigh anomaly (close to $850 \mathrm{~nm}$ ) are caused by a depolarization in the measured matrices coming from the finite spectral resolution of the monochromator of the polarimeter. It has a washing-out effect on sharp spectral features and introduces additional depolarization into the measured Mueller matrices [13] which leads to a lack of accuracy in the retrieved matrix.

Overall performance of the numerical approach demonstrated in comparison of the reconstructed and directly measured Mueller matrices of the grating in Fig. 8 (blue and black curves) is promising for applications, where beam spot size is larger than the sample. Using this method, which can filter one of components from depolarizing matrix measurements, can lead to a strong economical advantage as compared to the necessity to develop or buy new characterization tool. This will be especially true if the size of the sample is comparable with the spot size of some already existing tool.
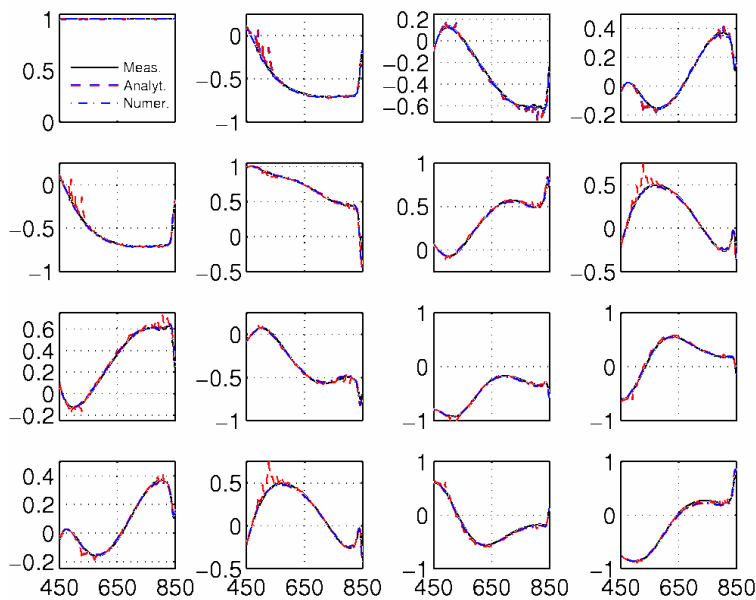

Fig. 8. Spectral values of the analytically (dashed red line) and numerically (dash-dotted blue line) retrieved
Mueller matrices compared with the directly measured matrix of the grating (solid black line).

\section{Conclusions}

In this work we have outlined applications of the Mueller matrix polarimetry to the grating profile characterization and advantages of using conical configurations as compared with standard planar configuration. Potential of the Mueller matrix polarimetry in angle-resolved, spectral or combined configurations has been shown on particular examples of etched photoresist and silicon gratings. New approach allowing estimating confidence intervals for critical dimensions based on the multi-azimuth method was presented in Section 3. Further prospect of using rich information in Mueller matrices in order to distinguish different non-depolarizing components of depolarizing matrices has been shown. An application of the method has been presented on the measurements of samples smaller that the beam spot size.

\section{References}

1. B. Bunday, A. Peterson, J. Allgair, Proc SPIE 5752, 304 (2005).

2. V. A. Ukraintsev, C. Baum, G.Zhang, C. L. Hall, Proc SPIE 5752, 127 (2005).

3. J. Foucher, E. Pargon, M. Martin, S. Reyne, C. Dupré, Proc SPIE 6922, 69220F (2008).

4. V.A. Ukraintsev, Proc. SPIE 6152, $61521 \mathrm{G}$ (2006).

5. A. De Martino, M. Foldyna, T. Novikova, D. Cattelan, P. Barritault, C. Licitra, J. Hazart, J. Foucher, F. Bogeat, Proc. SPIE 6922, 69221P (2008).

6. M. Foldyna, A. De Martino, D. Cattelan , F. Bogeat, C. Licitra, J. Foucher, P. Barritault, J. Hazart, Proc. SPIE 7140, 71400I (2008).

7. E. Compain, S. Poirier, B. Drévillon, Appl. Opt. 38 3490 (1999).

8. A. De Martino, S. Ben Hatit, M. Foldyna, Proc SPIE 6518, 65180X (2007).

9. S. Ben Hatit, M. Foldyna, A. De Martino, B. Drévillon, Phys. Stat. Sol. A 205, 743 (2008).

10. M. Foldyna, A. De Martino, C. Licitra, J. Foucher, Proc SPIE 7638, accepted.

11. J. M. Correas, P. Melero, J. J. Gil, Mon. Sem. Mat. García de Galdeano 27, 233 (2003).

12. J. J. Gil, Eur. Phys. J. Appl. Phys. 40, 1 (2007).

13. M. Foldyna, A. De Martino, R. Ossikovski, E. Garcia-Caurel, C. Licitra, Opt. Commun. 282, 735 (2009).

14. M. Foldyna, E. Garcia-Caurel, R. Ossikovski, A. De Martino, J. J. Gil, Opt. Express 17, 12794 (2009). 